\title{
OP79
}

\section{PHOTOMETER SPECTRAL RESPONSE MEASUREMENT USING OPO TUNABLE LASER}

\author{
Weiqiang Zhao et al. \\ DOI 10.25039/x46.2019.OP79 \\ from \\ CIE x046:2019 \\ Proceedings \\ of the \\ 29th CIE SESSION \\ Washington D.C., USA, June 14 - 22, 2019 \\ (DOI 10.25039/x46.2019)
}

The paper has been presented at the 29th CIE Session, Washington D.C., USA, June 14-22, 2019. It has not been peer-reviewed by CIE.

(C) CIE 2019

All rights reserved. Unless otherwise specified, no part of this publication may be reproduced or utilized in any form or by any means, electronic or mechanical, including photocopying and microfilm, without permission in writing from CIE Central Bureau at the address below. Any mention of organizations or products does not imply endorsement by the CIE.

This paper is made available open access for individual use. However, in all other cases all rights are reserved unless explicit permission is sought from and given by the $\mathrm{CIE}$.

CIE Central Bureau

Babenbergerstrasse 9

A-1010 Vienna

Austria

Tel.: +43 17143187

e-mail: ciecb@cie.co.at

www.cie.co.at 


\title{
PHOTOMETER SPECTRAL RESPONSE MEASUREMENT USING OPO TUNABLE LASER
}

\author{
Zhao, W.Q., Liu, H., Yan, J.Y., Su, Y., Jiang, L., Wu, Z.F. \\ National Institute of Metrology, CHINA \\ zhaowq@nim.ac.cn
}

DOI $10.25039 / \times 46.2019 .0 P 79$

\begin{abstract}
This paper shows an photometer spectral response measurement method using an pulsed tunable laser. The laser is the comparator. the standard detector is a three-silicon S1337 trap detector and the detector under test is a silicon photodiode with a $V(\lambda)$-correction filter. Due to the low noise and strong signal of the laser, we believe it will give a lower measurement uncertainty compared to traditional method for measuring $S(\lambda)$ of the photometer
\end{abstract}

Keywords: Photometer, Spectral response, Pulsed tunable laser,Trap detector

\section{Introduction}

Nowadays, LED and related products are widely used in everyday lighting. It is important to the get precise photometric measurement results for LED.For colour LED photometric measurement, one of the significant measurement uncertainties comes from colour correction factor $(C C F) C_{\text {ccf, }}$ when the relative spectral response of the photometer $S^{*}(\lambda)$ is different from the photopic luminous efficiency function $V(\lambda)$. For example, to test an LED average luminous intensity using an photometer, which is calibrated by using CIE A illuminant as light source. The CCF is determined by $S^{*}(\lambda), V(\lambda)$, spectra of measurand $P_{\operatorname{LED}}(\lambda)$ and spectra of CIE A illuminant $P_{\mathrm{CIE}} \mathrm{A}(\lambda)$. The CCF is defined as the following expression (1)

$$
C_{\mathrm{ccf}}=\frac{\int_{380}^{780} P_{\mathrm{LED}}(\lambda) V(\lambda) \mathrm{d} \lambda}{\int_{380}^{780} P_{\mathrm{CIE} \mathrm{A}}(\lambda) V(\lambda) \mathrm{d} \lambda} \frac{\int_{380}^{780} P_{\mathrm{CIEA}}(\lambda) S^{*}(\lambda) \mathrm{d} \lambda}{\int_{380}^{780} P_{\mathrm{LED}}(\lambda) S^{*}(\lambda) \mathrm{d} \lambda}
$$

Therefore, an accuracy $S^{*}(\lambda)$ measurement result of the photometer helps to get a low uncertainty CCF. That means lower measurement uncertainty of LED photometric measurement, especially for blue LEDs.

\section{Method and Setup}

The pulsed optical parametric oscillator (OPO) tunable laser of a $1 \mathrm{kHz}$ repetition frequency system, which covers the range of $210 \mathrm{~nm} \sim 2400 \mathrm{~nm}$ spectrum, was used to calibrate silicon photodiode with a low measurement uncertainty in a previous report [1]. Great potential in field of photometry and radiometry is shown. In this report, we have recently setup a photometer spectral response measurement system using an pulsed OPO tunable laser. The tunable laser system has a pulse laser of $1 \mathrm{k} \mathrm{Hz}$ repetition frequency and each pulse has a $\sim 5 \mathrm{~ns}$ pulse width. In the visible range of $405 \mathrm{~nm} \sim 780 \mathrm{~nm}$, the width at half maximum of the pulse spectra is smaller than $0.2 \mathrm{~nm}$. The facility is fully automated, easy to maintain and friendly to use.

After coming out of OPO, the laser light beam is coupled into a fibre and passes through the ultrasound bath, and then inject into an integrating sphere. The aperture of the integrating sphere is the light source for the measure system.

To measure $S^{*}(\lambda)$ of the photometer, the detector under test (DUT) is made of a silicon photodiode with a $V(\lambda)$-correction filter. The standard detector (STD) is a three-silicon S1337 trap detector. The substitution method is used when DUT and STD are mounted on motorized 
stage. Because the pulse to pulse is variation and not easy to stabilize, monitor detector (MD) is need, and be fixed with the same distance to the output integrating sphere as the DUT/STD does, with a different angle. Baffles and light traps are used to contain the stray light. The schematic is shown in figure 1.

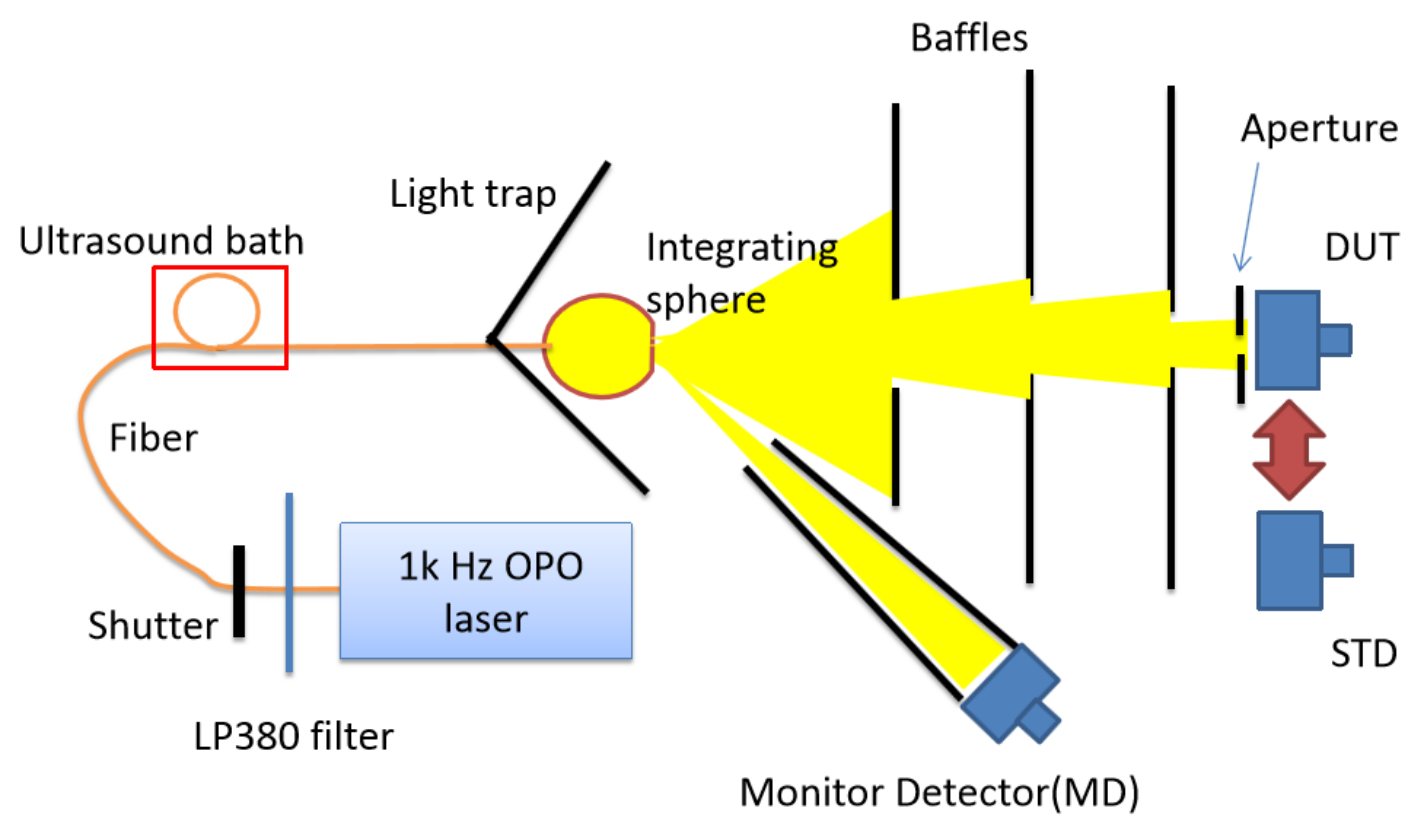

Figure 1 - The schematic setup for photometer spectral response measurement

The response of DUT $S_{\text {DUT }}(\lambda)$ is measured using the expression (2). Here, $S_{\mathrm{STD}}(\lambda)$ is the spectral response of the STD, $R_{\mathrm{DU} T}(\lambda)$ is reading of DUT, $R_{\mathrm{MD}, \mathrm{DU} T}(\lambda)$ is the reading of $\mathrm{MD}$ while DUT is on , $R_{\mathrm{STD}}(\lambda)$ is reading of STD, $R_{\mathrm{MD}, \mathrm{STD}}(\lambda)$ is the reading of MD while STD is on.

$$
S_{\mathrm{DUT}}(\lambda)=\frac{R_{\mathrm{DUT}}(\lambda)}{R_{\mathrm{MD}, \mathrm{DUT}}(\lambda)} \cdot \frac{R_{\mathrm{MD}, \mathrm{STD}}(\lambda)}{R_{\mathrm{STD}}(\lambda)} \cdot S_{\mathrm{STD}}(\lambda)
$$

Then $S^{*}(\lambda)$ is normalized result of $S_{\mathrm{DUT}}(\lambda)$.

The reading of DUT,STD and MD is measured by electrometers, and same method in report[1].

\section{Results}

We test one photometer spectral response range from $405 \mathrm{~nm}$ to $780 \mathrm{~nm}$. The relative standard deviation (RSD) of the measurement are shown in figure 1. 


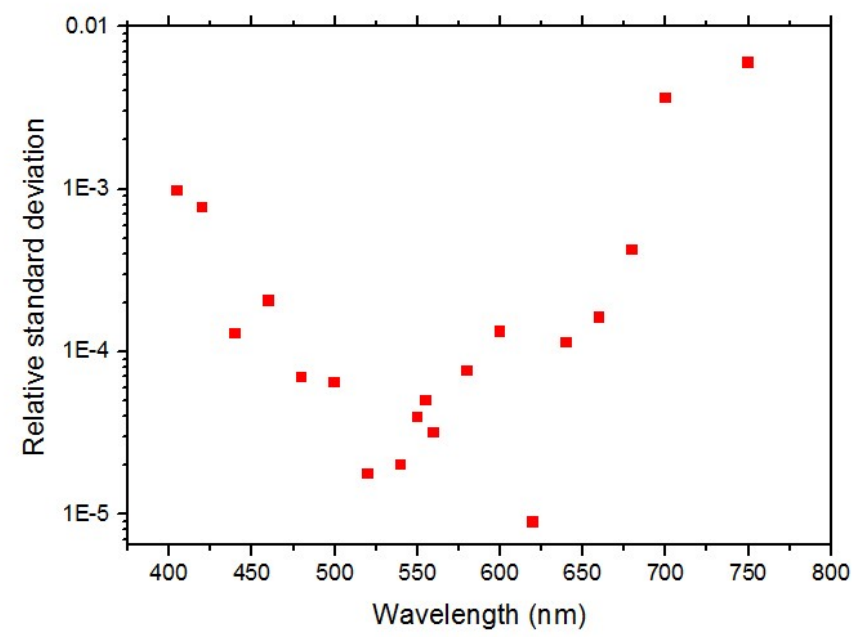

Figure 2 - The relative standard deviation of the measurement

The RSD is less the $0.01 \%$ in the range of $460 \mathrm{~nm} \sim 580 \mathrm{~nm}$, and is $\sim 0.01 \%$ in the range of 440 $\mathrm{nm} \sim 460 \mathrm{~nm}$ and $580 \mathrm{~nm} \sim 660 \mathrm{~nm}$. RSD increases in the violet zone the deep red zone, due to the weak output signal of the photometer.

For most of blue LED, the wavelength of spectra peak is of $\sim 450 \mathrm{~nm}$. The measurement results shows a good signal to noise ratio (> 5000:1) and good repeatability at $450 \mathrm{~nm}$. Uncertainty evaluation is still on-going, however we believed it will give a lower number compared to traditional method for measuring $S^{*}(\lambda)$ of the photometer. And the photometer spectral response measurement results are shown in figure 3.

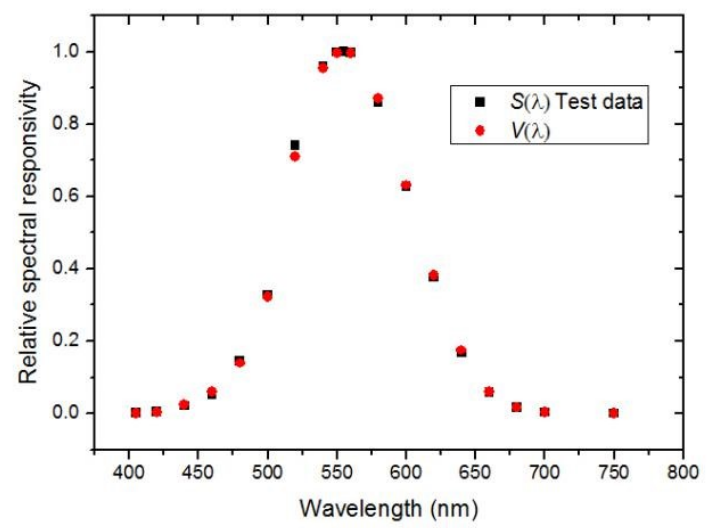

Figure 3 - The photometer spectral response measurement result

The uncertainties evaluation is still on-going,however, however we show a rough estimated results in table 1. 
Table 1 - Rough estimated uncertainties.

\begin{tabular}{|c|c|c|}
\hline & \multicolumn{2}{|c|}{ Relative standard uncertainty (\%) } \\
\hline Uncertainty component & $450 \mathrm{~nm}$ & $555 \mathrm{~nm}$ \\
\hline STD trap & 0.06 & 0.06 \\
\hline Laser wavelength & 0.05 & 0.02 \\
\hline Irradiance uniformity & 0.05 & 0.05 \\
\hline Detector linearity & 0.005 & 0.005 \\
\hline Electrometer linearity & 0.02 & 0.02 \\
\hline Repeatability & 0.02 & 0.01 \\
\hline Combined Uncertainty & 0.10 & 0.08 \\
\hline$U_{\text {rel }} \quad(\mathrm{k}=2)$ & 0.2 & 0.16 \\
\hline
\end{tabular}

\section{Conclusion}

This paper shows an photometer spectral response measurement method using an OPO tunable laser. Due to the low noise and strong signal of the laser, we believe it will give a lower measurement uncertainty compared to traditional method for measuring $S(\lambda)$ of the photometer. An rough estimated measurement uncertainty at $450 \mathrm{~nm}$ is $U_{\mathrm{rel}}=0.2 \%(k=2)$.

\section{Reference}

[1] Yuqin Zong etc., New Method for Spectral Irradiance and Radiance Responsivity Calibration using Pulsed Tunable Lasers, NEWRAD 2011 Maui,Hawaii,USA 